\title{
Individual- and Neighbourhood-Level Indicators of Subjective Well-Being in a Small and Poor Eastern Cape Township: The Effect of Health, Social Capital, Marital Status, and Income
}

\author{
J. M. Cramm • V. Møller • A. P. Nieboer
}

Accepted: 18 September 2010/Published online: 20 January 2011

(C) The Author(s) 2011. This article is published with open access at Springerlink.com

\begin{abstract}
Our study used multilevel regression analysis to identify individual- and neighbourhood-level factors that determine individual-level subjective well-being in Rhini, a deprived suburb of Grahamstown in the Eastern Cape province of South Africa. The Townsend index and Gini coefficient were used to investigate whether contextual neighbourhood-level differences in socioeconomic status determined individual-level subjective well-being. Crime experience, health status, social capital, and demographic variables were assessed at the individual level. The indicators of subjective well-being were estimated with a two-level random-intercepts and fixed slopes model. Social capital, health and marital status (all $p<.001)$, followed by income level $(p<.01)$ and the Townsend score $(p<.05)$ were significantly related to individual-level subjective well-being outcomes. Our findings showed that individual-level subjective well-being is influenced by neighbourhood-level socioeconomic status as measured by the Townsend deprivation score. Individuals reported higher levels of subjective well-being in less deprived neighbourhoods. Here we wish to highlight the role of context for subjective well-being, and to suggest that subjective well-being outcomes may also be defined in ecological terms. We hope the findings are useful for implementing programs and interventions designed to achieve greater subjective well-being for people living in deprived areas.
\end{abstract}

Keywords Multilevel regression analysis - Neighbourhood socioeconomic status · Deprived communities · Subjective well-being $\cdot$ South Africa

J. M. Cramm $(\bowtie) \cdot$ A. P. Nieboer

Institute of Health Policy \& Management (iBMG), Erasmus University Rotterdam,

Burgemeester Oudlaan 50, 3062 PA Rotterdam, The Netherlands

e-mail: cramm@bmg.eur.nl

A. P. Nieboer

e-mail: nieboer@bmg.eur.nl

V. Møller

Rhodes University of Grahamstown, Grahamstown, South Africa

e-mail: v.moller@ru.ac.za 


\section{Introduction}

In recent years, scholars in a wide range of disciplines have attempted to define, measure and analyze well-being in various contexts (Hoorn 2007). A recent study defines subjective well-being as "an umbrella term for different valuations that people make regarding their lives, the events happening to them, their body and minds, and circumstances in which they live" (Diener 2006). While happiness cannot be directly measured, the validity and reliability of several measures of subjective well-being have been demonstrated (Pavot and Diener 1993). Questions concerning well-being are increasingly used in population surveys, reflecting the emergence of a new science of well-being. This pursuit aims to identify the main factors affecting well-being and to quantify their relative importance. There is a rapidly growing body of interdisciplinary research on subjective well-being (Dolan et al. 2008; Frey and Stutzer 2002).

Evidence suggests that subjective well-being may fuel individual differences in resilience over time (Fredrickson 2001; Fredrickson and Joiner 2002; Lyubomirsky et al. 2005). The positive effects of enhanced subjective well-being on resilience can be explained by an individual's personal resources such as physical health, economic activity, and social contacts (Isen 1990; Aspinwall 1998, 2001; Fredrickson 2004). Importantly, these resources function as buffers, enabling individuals to achieve well-being in multiple ways because they can substitute one resource for another (Nieboer and Lindenberg 2002; Nieboer et al. 2005). The risk of low subjective well-being is much higher for poor people (Diener and Biswas-Diener 2002; Cramm et al. 2010). Better-off individuals can substitute one subjective well-being resource for another; small changes for poor people who lack these resources often negatively impact their well-being levels (Diener and Lucas 2000; Nieboer and Lindenberg 2002; Nieboer et al. 2005). In this regard, it would be helpful to identify indicators for subjective well-being within poor communities. Such knowledge will enable to set up programs and interventions aimed at helping communities achieve greater subjective well-being for a greater number of people.

Most quantitative studies have used single-level regression models to infer relationships between well-being and a wide range of socioeconomic and demographic characteristics (Blanchflower and Oswald 2004) or to compare aggregate well-being levels between countries (Farid and Lazarus 2008; Marks et al. 2006; Veenhoven 1993, 2000). The influence of grouping factors like neighbourhoods, however, should also be taken into account. A systematic review of multilevel regression analyses (MLRA) of health outcomes has demonstrated consistent evidence for neighbourhood effects on health (Picket and Pearl 2001). Socioeconomic neighbourhood characteristics may also affect well-being between individuals (Deneulin and Townsend 2007). If individual well-being correlates with a shared environment, single-level regression analyses will underestimate the standard errors for contextual effects and produce biased results (Merlo et al. 2005). MLRA, however, can be used to identify the distribution of disparities in well-being at both individual and neighbourhood levels.

The influence of neighbourhood-level effects on individual well-being is probably greater among people living in socioeconomically deprived neighbourhoods. Spatial variations in economic and social conditions that produce concentrated deprivation are not merely reducible to "compositional effects" (i.e., the personal and family characteristics of the neighbourhood's population) and can involve a contextual neighbourhood-related effect (e.g., income inequality within neighbourhoods and the neighbourhood's socioeconomic status) resulting from concentrated disadvantage. The already disadvantaged people living in deprived neighbourhoods and dealing with poverty, (infectious) diseases, and the like are even more burdened with negative neighbourhood effects that compound 
problems of concentrated disadvantage and social exclusion. The effects of multilevel characteristics on individual well-being and the extent to which each indicator at each level affects individual well-being are currently unknown. Identifying multilevel factors that affect subjective well-being would help toward finding the most effective level of intervention and enable policymakers to design programs that achieve greater subjective wellbeing for a greater number of people.

This study seeks to identify indicators of subjective well-being at the individual and neighbourhood levels in a community in the Eastern Cape province of South Africa characterized by high levels of poverty and unemployment (Møller and Erstad 2007) and multiple deprivation domains, such as income, employment, health, and education (Noble et al. 2009). Subjective well-being has not been thoroughly examined at lower levels of aggregation, such as neighbourhoods. A smaller spatial unit of analysis and consistent economic deprivation may dramatically affect the variables that contribute to subjective well-being. It remains unclear whether contextual factors will continue to account for differences in subjective well-being at smaller spatial units of analysis characterised by consistent deprivation.

South Africa's historical pattern of racial segregation may have led to low levels of subjective well-being in some settings (mostly townships). The mean well-being score (0-10) for the community under study is 4.6 (Møller 2007b), which is substantially lower than found in South Africa as a whole (5.7) (Marks et al. 2006). Since enhanced subjective well-being shows positive effects on health as well as social and economic activity, the population suits the purposes of our study.

\subsection{Contextual Neighbourhood-Level Indicators of Individual Subjective Well-Being}

Absolute level of income is not the only relevant economic variable Wilkinson and Pickett (2006) have concluded that subjective well-being tends to be lower in societies where relative income differences are larger. The degree of correlation substantially differed whether measured in large or small communities. The authors therefore have suggested that studies of income inequality will find that subjective well-being is explained by greater variance in large neighbourhoods, where the variable measures the scale of social stratification or extent of hierarchy (Wilkinson and Pickett 2006). A variant on the argument that relative income influences well-being stems from the long-established literature on relative deprivation; individuals feel deprived if they are doing less well than their comparator (Knight and Song 2006). Relative poverty is likely to be more important in countries with pronounced economic inequality, but most research has reported results at high geographical levels of aggregation. Studies on communities within the same city are scarce (Wong et al. 2009).

The valid and reliable (Kawachi et al. 2003) Townsend deprivation index (Townsend et al. 1988) is widely used in academic health research (Galobardes et al. 2007). Since socioeconomic status affects health at the individual level, it may also affect subjective well-being. This has not been investigated, however, and further research is necessary to establish the association (Oswald 2007).

\subsection{Compositional Individual-Level Indicators of Subjective Well-Being}

Factors of demography, income, unemployment, health, and social capital are potential indicators of subjective well-being (Ahuvia and Friedman 1998; Andrews and Withey 1976; Campbell et al. 1976; Clark and Oswald 1994; Cramm et al. 2010; Diener et al. 1985, 1993; Larson 1978). Early research in this field tended to focus primarily on the relation of 
subjective well-being to demographic factors such as age, gender, education, and marital status. Such factors, however, have been shown to account for only a small amount of variance (Diener and Biswas-Diener 2002). Although some trends linking subjective wellbeing and age have been observed, the findings have not been consistently replicated and the effect appears to be small (Diener and Scollon 2003). Some researchers have identified a "u-shaped" relationship between age and well-being, suggesting that individuals are happier in youth and old age (Blanchflower and Oswald 2004; Gerdtham and Johannesson 2001; Hoorn 2007). Some studies examining gender differences in well-being have reported slightly higher subjective well-being in females than in males (Frey and Stutzer 2002; Gerdtham and Johannesson 2001; Hoorn 2007). Other studies, however, have found no gender differences (Diener and Scollon 2003) or argue that they have disappeared in recent decades (Frey and Stutzer 2002). Research has found that married people, on average, are happier than unmarried people (Diener and Scollon 2003; Frey and Stutzer 2002; Hoorn 2007). Evidence for the impact of education on subjective well-being has been mixed. Some studies suggest a positive relationship (Diener and Scollon 2003; Frey and Stutzer 2003), while others (Clark and Oswald 1994) have observed a negative impact that may be due to changing aspirations and the creation of expectations for a higher income.

Extensive research has been conducted on the relationship between economic variables and well-being, revealing that subjective well-being correlates positively with income (Cramm et al. 2010; Fahey et al. 2005; Keck and Krause 2007). Most studies report a positive but relatively weak relationship between absolute income and happiness (Cramm et al. 2010; Diener and Biswas-Diener 2002; Easterlin 1974). Unemployment shows a consistently negative association with well-being and may have severe long-term impacts (Clark and Oswald 1994; Hoorn 2007).

Studies consistently report a high positive correlation between well-being and health (Diener 2002; Diener and Scollon 2003; Dolan et al. 2008; Frey and Stutzer 2002; Hoorn 2007). Research indicates that individuals reporting higher levels of subjective well-being live longer than their counterparts. Good health is probably both a cause and an effect of high levels of well-being (Diener and Biswas-Diener 2002).

Social capital is also increasingly acknowledged to be an important indicator of subjective well-being (Bjørnskov 2003, 2005; Cramm et al. 2010; Easterlin 2000; Grootaert 2002; Wilkinson and Pickett 2006; Yip et al. 2007; Haggerty et al. 2001). It is defined as "the set of cooperative relationships between social actors that facilitate collective actions" (Requena 2003) and its core components are civic engagement and mutual trust among community members. Social capital shelters people from the harmful effects of unemployment and poverty (Camfield and Skevington 2008; Cramm et al. 2010; Winkelman 2009). Various studies have proposed that people with friendly, helpful, and trustworthy neighbours report higher levels of subjective well-being than those who are more solitary (Wilkinson and Pickett 2006). Marks et al. (2006) have indicated a strong relationship between greater social capital and higher life satisfaction. Socio-cultural features such as crime have also been found to be associated with well-being (Borooah 2006; Chan and Lee 2006).

\section{Methods}

\subsection{Participants and Sampling}

This study applied a neighbourhood-stratified sampling design. Households in Rhini, a suburb of Grahamstown, South Africa, were randomly selected in proportion to the total 
number of households in each neighbourhood. In each of the 20 neighbourhoods of Rhini, a random starting point was taken. Moving systematically through the neighbourhood, the researchers selected every tenth household for inclusion in the sample. This method ensured that all households in all neighbourhoods of Rhini stood an equal chance of being included in the survey.

Eligible respondents identified in each target household were at least 18 years old and had resided in Rhini for at least 6 months of the past year. One respondent per randomlyselected household was selected using a Kish grid (to ensure all eligible persons in the household stood an equal chance of being included in the survey) and interviewed. If this person was not available, up to four attempts were made to conduct the interview at a later time. The interview rate was 1,020 of 1,042 targeted households (97.9\%). Reasons for forgoing the interview included not finding the respondent at home after four visits, old age or poor health, and disinterest or unwillingness.

Staff from Development Research Africa, a well-known organization experienced in undertaking national probability-based samples in deep rural and urban areas, administered the questionnaires. Almost all questions were closed-ended and a set of response options was supplied. The interviewers gathered demographic information about the participants, such as gender, age, health status, education level, living arrangements, and employment. A detailed description of this study population can be found in Møller (2007a, 2008).

\subsection{Areas}

Housing, infrastructure, and access to services in Grahamstown East/Rhini are historically grounded. The period of development is telling in terms of housing and neighbourhood conditions (Møller 2007a). Each neighbourhood has had its own wave of solutions to modernization, which may have contributed to differences in individual subjective wellbeing outcomes.

\subsection{Measurements}

Diener's Satisfaction with Life Scale (SWLS) was used to assess respondents' satisfaction with life as a whole. This instrument consists of five items rated on a 5-point scale: (1) in most ways my life is close to my ideal; (2) the conditions of my life are excellent; (3) I am satisfied with my life; (4) so far I have gotten the important things I want in life; and (5) if I could live my life over, I would change almost nothing (Pavot and Diener 1993). Cronbach's alpha for the SWLS in the present study was 0.88 , indicating that the scale was reliable.

The Townsend index was used to investigate neighbourhood-level socioeconomic status effects on individual-level subjective well-being. It includes four variables: unemployment (lack of material resources and insecurity); overcrowding (material living conditions); lack of owner-occupied accommodation (a proxy indicator of wealth); and lack of car ownership (a proxy indicator of income). The four variables combine to form an overall score, which is a summation of the standardized scores ( $\mathrm{z}$ scores) for each variable (scores $>0$ indicate greater levels of material deprivation). A higher Townsend index score indicates a more deprived and disadvantaged neighbourhood. Since the score is considered the most reliable and viable indicator of material deprivation available, we used it to investigate neighbourhood-level differences in socioeconomic status and individual-level subjective well-being (Galobardes et al. 2007; Townsend et al. 1988). 
The Gini coefficient of inequality was used to measure income inequality. The coefficient ranges from 0 (complete equality) to 1 (complete inequality) and has been shown to be valid and reliable (Kawachi et al. 1997).

Crime experience was assessed with the statement, "There is not a lot of crime in this neighbourhood". Respondents rated their level of agreement on a four-point scale.

Health status was measured via self-rated health, which is considered a valid and robust measure (Wen et al. 2003). A large body of evidence has demonstrated that self-reported health assessment has high predictive validity for mortality, physical disability, and chronic disease status. Furthermore, self-assessed health is a stronger predictor of mortality than physician-assessed health (Idler and Benyamanini 1997; Idler and Kasl 1995; Mossey and Shapiro 1982). As a subjective measure of health status, this measure captures the personally experienced problems of physical well-being that may impair subjective wellbeing (Wen et al. 2003).

Social capital was assessed with three items: "People in this neighbourhood are (1) friendly; (2) help each other without having to be asked; (3) trust their neighbours", to which respondents rated their level of agreement on a four-point scale. Cronbach's alpha was 0.87 . The questionnaire further probed factors previously implicated as potential indicators of subjective well-being, such as income, education, unemployment, marital status, gender and age.

\subsection{Data Analysis}

Our data analysis sought to verify the impact of contextual neighbourhood- and compositional individual-level effects on individual subjective well-being. We generated descriptive summary statistics and used Spearman's rank correlations to explore univariate associations between the independent variables and subjective well-being. To account for the hierarchical structure of the study design we fitted a hierarchical random-effects model. The hierarchical structure comprises 1,020 individuals (level 1) nested in 20 neighbourhoods (level 2) of Rhini. Individuals were excluded if observations were missing for any outcome, leading to a total of 957 individuals in the MLRA. The independent variables were all standardized. The indicators of subjective well-being were estimated with a twolevel random-intercepts and fixed slopes model structure. The resulting estimated parameters in the fixed part were tested by dividing the regression coefficient by its standard error. All regression models were employed using SPSS (Version 17.0) software.

We report our results in the sequence of analysis. To estimate the relative contributions of independent variables, we calculated the reduction in model deviance from the null (intercept only) to models containing the intercept and each individual variable. To estimate the strength of associations we obtained explained variance at both individual and neighbourhood levels. First we described the estimates of the empty model (1), which reflects variation in the intercept. Second, we estimated the adjusted coefficients of the different independent variables in two series of models (models 2 and 3). In model 2 we added the two contextual indicators at neighbourhood-level (the Townsend score and Gini coefficient). Because it is unclear whether contextual factors will continue to account for differences in subjective well-being at smaller spatial units of analysis characterised by consistent deprivation, we first added the contextual indicators (Gini coefficient and Townsend score) in model 2. Model 3 contained the adjusted compositional coefficients of crime experience, social capital and health, the economic indicators of unemployment and income, and demographic data (age, gender, marital status and education). 


\section{Results}

Respondents had a median age of 38 (range $=18-98$ ) and the majority was female $(73 \%)$. Just over half were single (52\%), a third married (33\%), and the others widowed (9\%) or separated/divorced (6\%). Forty percent had completed some secondary education and $18 \%$ had matriculated. Approximately $7 \%$ had received post-matriculation education and training. Only $8 \%$ had no formal schooling. Respondents' unemployment rate was $62 \%$. Tables 1 and 2 provide descriptive statistics for the other individual-level independent variables and neighbourhood-level information, respectively.

Univariate analyses of the associations between the neighbourhood- and individuallevel indicators and subjective well-being are presented in Table 3. The Townsend score, social capital, health, unemployment, income, marital status and education were all significantly correlated with subjective well-being in our sample.

The MLRA simultaneously evaluated the effects of neighbourhood-level indicators (Townsend score and Gini coefficient) and individual-level indicators (crime experience, social capital, health status, unemployment, income, and demographics) on subjective well-being at the individual level. Table 4 presents the results of MLRA. The first set of contextual indicators documents the influences of neighbourhood-level socioeconomic status (Townsend score) and neighbourhood-level income inequality (Gini coefficient) on individuals' subjective well-being. They were tested by regressing subjective well-being on possible indicators of subjective well-being (model 2 of Table 4). Neighbourhood-level socioeconomic status appears to be a significant indicator of subjective well-being (Townsend score: $\beta-.118 ; p<.05$ ), while neighbourhood-level income inequality is not. In sum, individuals appear to have higher reported levels of subjective well-being when their neighbourhood is less deprived when measured with the Townsend deprivation score. Income inequality within the neighbourhood was less influential for subjective well-being outcomes in our population. The explained neighbourhood-level variance is $30 \%$.

The second set of indicators measured the association between crime experience, social capital, health, unemployment, income, demographics and subjective well-being by regressing subjective well-being on the indicators (model 3 of Table 4). The Townsend score remains an indicator of subjective well-being $(\beta-.110 ; p<.05)$. Social capital is associated with subjective well-being $(\beta .138 ; p<.001)$ as is health status $(\beta .138$; $p<.001)$ and income level $(\beta .075 ; p<.01)$. Unemployment and neighbourhood crime did not emerge as significant indicators for subjective well-being. Among the demographic variables, only marital status was a strong indicator of subjective well-being $(\beta$.092; $p<.001)$. The explained variance is $8.3 \%$ at the individual level and $40.0 \%$ at the neighbourhood level. The strongest associations with subjective well-being in this population are social capital, health, and marital status (all $p<.001$ ), followed by income level $(p<.01)$ and the Townsend score $(p<.05)$.

Table 1 Descriptive statistics for the variables used in the regression analyses $(\mathrm{N}=1,020)$

\begin{tabular}{lllll}
\hline Model & Mean & SD & Min & Max \\
\hline Diener subjective well-being & 2.62 & 0.87 & 1.00 & 5.00 \\
Crime in the area & 2.98 & 0.89 & 1.00 & 4.00 \\
Social capital & 2.04 & 0.54 & 1.00 & 4.00 \\
Health status & 5.00 & 3.55 & 1.00 & 5.00 \\
Income & 5713 & 1863 & 0.00 & $>7001$ \\
\hline
\end{tabular}


Table 2 Summary descriptive statistics for neighbourhood-level indicators

\begin{tabular}{lrl}
\hline Neighbourhood & Townsend score & Gini coefficient \\
\hline 1 & 2.29 & 0.20 \\
2 & -0.66 & 0.17 \\
3 & -4.67 & 0.19 \\
4 & 0.39 & 0.19 \\
5 & -0.47 & 0.19 \\
6 & -2.94 & 0.18 \\
7 & -2.24 & 0.16 \\
8 & -8.27 & 0.16 \\
9 & -0.09 & 0.17 \\
10 & -0.08 & 0.21 \\
11 & 0.12 & 0.23 \\
12 & 0.74 & 0.18 \\
13 & 3.44 & 0.25 \\
14 & 1.29 & 0.19 \\
15 & 1.97 & 0.22 \\
16 & 1.21 & 0.21 \\
17 & 1.40 & 0.14 \\
18 & 1.42 & 0.24 \\
19 & 2.53 & 0.24 \\
20 & 0.47 & 0.20 \\
\hline
\end{tabular}

Table 3 Correlations between independent variables and subjective well-being

\begin{tabular}{lcc}
\hline $\mathrm{N}=1,020$ in 20 neighbourhoods & $r$ & $p$ \\
\hline Townsend score & -.112 & $<.0001$ \\
Gini coefficient & -.029 & .360 \\
Crime in the area & .003 & .919 \\
Social capital & .153 & $<.0001$ \\
Health status & .163 & $<.0001$ \\
Unemployment & -.147 & $<.0001$ \\
Income & .172 & $<.0001$ \\
Age & -.030 & .337 \\
Gender & -.039 & .221 \\
Marital status & .125 & $<.0001$ \\
Education level & .116 & $<.0001$ \\
\hline
\end{tabular}

\section{Discussion}

This article has presented cross-sectional evidence for the correlated nature of subjective well-being at the individual and neighbourhood levels. The key findings of this study are as follows. First, the Townsend deprivation score is a statistically significant indicator of subjective well-being. People living in more deprived areas report lower subjective wellbeing. 
Table 4 Multilevel regression analyses on subjective well-being

\begin{tabular}{|c|c|c|c|c|c|c|}
\hline \multirow[t]{2}{*}{ Model } & \multicolumn{2}{|l|}{1} & \multicolumn{2}{|l|}{2} & \multicolumn{2}{|l|}{3} \\
\hline & $\beta$ & se & $\beta$ & se & $\beta$ & se \\
\hline Constant & 3.373 & .059 & 3.362 & .052 & 2.612 & .052 \\
\hline Townsend score & & & $-.118^{\dagger}$ & .059 & $-.110^{\dagger}$ & .060 \\
\hline Gini coefficient & & & -.002 & .058 & .019 & .058 \\
\hline Age & & & & & .039 & .033 \\
\hline Gender & & & & & .005 & .026 \\
\hline Marital status & & & & & $.093^{\ddagger}$ & .026 \\
\hline Education level & & & & & .030 & .031 \\
\hline Social capital & & & & & $.138^{\ddagger}$ & .027 \\
\hline Unemployment & & & & & -.031 & .031 \\
\hline Income & & & & & $.075^{*}$ & .031 \\
\hline Health status & & & & & $.138^{\ddagger}$ & .031 \\
\hline Crime in the area & & & & & -.004 & .027 \\
\hline$-2 \log$ likelihood & 2542 & & 2537 & & 2274 & \\
\hline Variance level 1 individual & $.700^{*}$ & .031 & $.700^{\ddagger}$ & .032 & $.614^{\ddagger}$ & .028 \\
\hline Variance level 2 area & $.050^{\dagger}$ & .023 & $.035^{\dagger}$ & .018 & $.036^{\dagger}$ & .017 \\
\hline Explained variance level 1 & & & $0 \%$ & & $8.3 \%$ & \\
\hline Explained variance level 2 & & & $30.0 \%$ & & $40.0 \%$ & \\
\hline
\end{tabular}

${ }^{\ddagger} p \leq .001 ; * p \leq .01 ;{ }^{\dagger} p \leq 0.05$

Neighbourhood income inequality was not found to be statistically significant indicators of subjective well-being. These differences may be related to the scale of analysis. For example, compared to the national level of inequality in South Africa (0.58; United Nations 2009) this study investigated income inequality at a lower geographical level of aggregation, within a single South African township. The Gini coefficients of the 20 neighbourhoods in our Eastern Cape suburb ranged from 0.14 to 0.25. Compared to South Africa's national level of inequality, the residents of Rhini shared equal (low) incomes. Investigation of a larger region with more pronounced neighbourhood income inequalities may produce different results.

Second, social capital, marital status, health and income were strongly associated with subjective well-being. Third, in contrast with previous single-level subjective well-being studies that have consistently found a negative association with unemployment (Clark and Oswald 1994; Hoorn 2007), the variable did not emerge as a statistically significant indicator in our MLRA. The effect, however, could be minimized by the Townsend deprivation score and the income variable. In our correlation analysis, unemployment did significantly correlate with subjective well-being. Fourth, the demographic indicators of educational level, age, and gender did not statistically significantly affect individual-level subjective well-being in our study. However, univariate regression analysis showed that education correlated with subjective well-being. The effect of education in the MLRA may also be partly minimized by income and the Townsend deprivation score.

Our research is not without limitations. The cross-sectional design hampered our ability to capture neighbourhood dynamics and draw causal inferences. Although it is not possible to determine the direction of the association, our results establish a significant association, 
an important step that prompts further study to identify directionality. We were unable to take into account the impact of residential changes and the cumulative effects of the socioeconomic environment over time. Our study was restricted to a single Eastern Cape suburb, perhaps limiting the applicability of our findings, but given the many areas with similar neighbourhood characteristics that exist in African developing countries, our study is likely to be applicable elsewhere. To our knowledge, we are the first to use MLRA in the investigation of subjective well-being. Our results therefore need confirmation, especially using data from similar African areas.

In conclusion, the study demonstrated high degrees of explained variance at neighbourhood level. We highlighted the role of context for subjective well-being, and suggested that subjective well-being outcomes may also be defined in ecological terms. We identified multilevel factors that are associated with subjective well-being for people living in health- and economically-deprived areas and believe the findings are useful for implementing programs and interventions designed to achieve greater subjective wellbeing. Research shows that subjective well-being fuels individual differences in resilience that affect health outcomes (Fredrickson 2001). The positive effects of enhanced subjective well-being on resilience help build an individual's personal resources. These resources allow people to achieve well-being in multiple ways, as they can substitute one resource for another (Nieboer and Lindenberg 2002; Nieboer et al. 2005). It is therefore important that programs aimed at one resource (e.g., economic development) in deprived areas are not implemented at the expense of another (e.g., social capital) that may ultimately harm overall well-being scores (Cramm et al. 2010). Subjective well-being promotes physical health and longevity and as such the indicators of subjective well-being found in this study need to be protected. Healthy people, in turn, are more productive in economic and social terms and thus contribute to overall economic and social development.

Acknowledgments This research project was funded by the South Africa Netherlands research Programme on Alternatives in Development (SANPAD). The views expressed in the paper are those of the authors.

Open Access This article is distributed under the terms of the Creative Commons Attribution Noncommercial License which permits any noncommercial use, distribution, and reproduction in any medium, provided the original author(s) and source are credited.

\section{References}

Ahuvia, A. C., \& Friedman, D. (1998). Income, consumption, and subjective well-being: Toward a composite macromarketing model. Journal of Macromarketing, 18, 153-168.

Andrews, F. M., \& Withey, S. B. (1976). Social indicators of well-being: America's perception of life quality. New York: Plenum.

Aspinwall, L. G. (1998). Rethinking the role of positive affect in self-regulation. Motivation and Emotion, $22,1-32$.

Aspinwall, L. G. (2001). Dealing with adversity: Self-regulation, coping, adaptation, and health. In A. Tesser \& N. Schwarz (Eds.), The Blackwell handbook of social psychology (Vol. 1, pp. 591-614). Malden, MA: Blackwell.

Bjørnskov, C. (2003). The happy few: Cross-country evidence on social capital and life satisfaction. Kyklos, $56(1), 3-16$.

Bjørnskov, C. (2005). The multiple facets of social capital. European Journal of Political Economy, 22, $22-40$.

Blanchflower, D. G., \& Oswald, A. J. (2004). Well-being over time in Britain and the USA. Journal of Public Economics, 88(7-8), 1359-1386. 
Borooah, V. (2006). What makes people happy? Some evidence from Northern Ireland. Journal of Happiness Studies, 7(4), 427-465.

Camfield, L., \& Skevington, S. M. (2008). On subjective well-being and quality of life. Journal of Health Psychology, 13, 764-775.

Campbell, A., Converse, P. E., \& Rodgers, W. L. (1976). The quality of American life. New York: Russell Sage.

Chan, Y., \& Lee, R. (2006). Network size, social support and happiness in later life: a comparative study of Beijing and Hong Kong. Journal of Happiness Studies, 7(1), 87-112.

Clark, A. E., \& Oswald, A. J. (1994). Unhappiness and unemployment. Economic Journal, 104, 648-659.

Cramm, J. M., Møller, V., Nieboer, A. P. (2010). Improving subjective well-being of the poor in the Eastern Cape. Journal of Health Psychology. doi:10.1177/1359105310367833.

Deneulin, S., \& Townsend, N. (2007). Public goods, global public goods and the common good. International Journal of Social Economic, 34(1/2), 19-36.

Diener, E. (2006). Guidelines for national indicators of subjective well-being and ill-being. Journal of Happiness Studies, 7(4), 397-404.

Diener, E., \& Biswas-Diener, R. (2002). Will money increase subjective well-being? Social Indicators Research, 57(2), 119-169.

Diener, E., Horwitz, J., \& Emmons, R. A. (1985). Happiness of the very wealthy. Social Indicators Research, 16, 263-274.

Diener, E., \& Lucas, R. E. (2000). Explaining differences in societal levels of happiness: Relative standards, need fulfillment, culture, and evaluation theory. Journal of Happiness Studies, 1(1), 41-78.

Diener, E., Sandvik, E., Seidlitz, L., \& Diener, M. (1993). The relationship between income and subjective well-being: Relative or absolute? Social Indicators Research, 28, 195-223.

Diener, E., \& Scollon, C. N. (2003). Subjective well-being is desirable, but not the summum bonum. Workshop on Well-Being: University of Minnesota.

Dolan, P., Peasgood, T., \& White, M. (2008). Do we really know what makes us happy? A review of the economic literature on the factors associated with subjective well-being. Journal of Economic Psychology, 29, 94-122.

Easterlin, R. A. (1974). Does economic growth improve the human lot? Some empirical evidence. In P. A. David \& M. W. Reder (Eds.), Nations and households in economic growth (pp. 89-125). New York: Academic Press.

Easterlin, R. A. (2000). Income and happiness: Towards a unified theory. The Economic Journal, 111(473), 465-484.

Fahey, T., Whelan, C. T., \& Maitre, B. (2005). First European quality of life survey: Income inequalities and deprivation. Luxembourg: Office for Official Publications of the European Communities.

Farid, M., \& Lazarus, H. (2008). Subjective well-being in rich and poor countries. Journal of Management Development, 27(10), 1053-1065.

Fredrickson, B. L. (2001). The role of positive emotions in positive psychology: The broaden-and-build theory of positive emotions. American Psychologist, 56, 218-226.

Fredrickson, B. L. (2004). The broaden-and-build theory of positive emotions. Philosophical Transactions of the Royal Society of London (Biological Sciences), 359, 1367-1377.

Fredrickson, B. L., \& Joiner, T. (2002). Positive emotions trigger upward spirals toward emotional wellbeing. Psychological Science, 13, 172-175.

Frey, B., \& Stutzer, A. (2002). Happiness and economics. Princeton: Princeton University Press.

Frey, B. S., \& Stutzer, A. (2003). Maximising happiness? German Economic Review, 1(2), 145-167. doi: 10.1111/1468-0475.00009.

Galobardes, B., Lynch, J., \& Davey Smith, G. (2007). Measuring socioeconomic position in health research. British Medical Bulletin, 2007, 1-17.

Gerdtham, U. G., \& Johannesson, M. (2001). The relationship between happiness, health and socio-economic factors: Results based on Swedish micro data. Journal of Socio-Economics, 30, 553-557.

Grootaert, C. (2002). Social capital, household welfare and poverty in Indonesia. Policy Research Working Paper no. 2148. Washington D.C.: The World Bank.

Haggerty, M. R., Cummins, R. A., Ferriss, A. L., Land, K., Michalos, A. C., Peterson, M., et al. (2001). Quality of life indexes for national policy: Review and agenda for research. Social Indicators Research, 55(1), 1-96.

Hoorn, A. (2007). A short introduction to subjective well-being: Its measurement, correlates and policy use. http://www.oecd.org/dataoecd/16/39/38331839.pdf. Accessed Sept 2009.

Idler, E. L., \& Benyamanini, Y. (1997). Self-rated health and mortality: A review of twenty-seven community studies. Journal of Health and Social Behavior, 38(1), 21-37. 
Idler, E. L., \& Kasl, S. V. (1995). Self-ratings of health: Do they predict change in functional ability? Journal of Gerontology: Social Sciences, 50(6), 344-353.

Isen, A. M. (1990). The influence of positive and negative affect on cognitive organization: Some implications for development. In N. Stein, B. Leventhal, \& T. Trabasso (Eds.), Psychological and biological approaches to emotion (pp. 75-94). Hillsdale, NJ: Erlbaum.

Kawachi, I., Berkman, L. F., \& Gordon, D. (2003). Area based deprivation measures: A U.K. perspective. In I. Kawachi \& L. F. Berkman (Eds.), Neighborhoods and health. New York: Oxford University Press.

Kawachi, I., Kennedy, B. P., Lochner, K., \& Prothrow-Stich, D. (1997). Social capital, income inequality, and mortality. American Journal of Public Health, 87, 1491-1498.

Keck, W., \& Krause, P. (2007). How does European Union enlargement affect social cohesion? In R. J. Estes (Ed.), Advancing quality of life in a turbulent world (pp. 3-24). Dordrecht: Springer.

Knight, J., \& Song, L. (2006). Subjective well-being and its determinants in rural China. mimeo: University of Nottingham.

Larson, R. (1978). Thirty years of research on the subjective well-being of older Americans. Journal of Gerontology, 33, 109-125.

Lyubomirsky, S., King, L. A., \& Diener, E. (2005). The benefits of frequent positive affect: Does happiness lead to success? Psychological Bulletin, 131, 803-855.

Marks, N., Abdallah, S., Simms, A., \& Thompson, S. (2006). The happy planet index. London: New Economics Foundation.

Merlo, J., Yang, M., \& Chair, B. (2005). A brief conceptual tutorial on multilevel analysis in social epidemiology: Investigating contextual phenomena in different groups of people. Journal of Epidemiology and Community Health, 29, 443-449.

Møller, V. (2007a). Living in Rhini. A 2007 update on the 1999 social indicators report. Institute of Social and Economic Research, Research Report Series No. 14.

Møller, V. (2007b). Researching quality of life in a developing country: Lessons from the South Africa case. In I. Gough \& J. A. McGregor (Eds.), Wellbeing in developing countries: From theory to research (pp. 242-258). Cambridge: Cambridge University Press.

Møller, V. (2007c). Satisfied and dissatisfied South Africans: Results from the General Household Survey in international comparison. Social Indicator Research, 81(2), 389-415.

Møller, V. (2008). Destigmatising TB in the time of HIV/AIDS. Attitudes, beliefs, and recommendations from an Eastern Cape community. Institute of Social and Economic Research, Research Report Series No. 15.

Møller, V., \& Erstad, I. (2007). Stigma associated with tuberculosis in a time of HIV/AIDS: Narratives from the Eastern Cape, South Africa. South African Review of Sociology, 38, 103-119.

Mossey, J. M., \& Shapiro, E. (1982). Self-rated health: A predictor of mortality among the elderly. American Journal of Public Health, 72(8), 800-808.

Nieboer, A., \& Lindenberg, S. (2002). Substitution, buffers and subjective well-being: A hierarchical approach. In E. Gullone \& R. A. Cummins (Eds.), The universality of subjective well-being indicators (pp. 175-189). Dordrecht, Netherlands: Kluwer Academic Publishers.

Nieboer, A., Lindenberg, S., Boomsma, A., \& van Bruggen, A. C. (2005). Dimensions of well-being and their measurement: The SPF-IL scale. Social Indicator Research, 73, 313-353.

Noble, M., Barnes, H., Wright, G., \& Roberts, B. (2009). Small area indices of multiple deprivation in South Africa. Social Indicator Research., 95(2), 281-297.

Oswald, A. (2007). Commentary: Human well-being and causality in social epidemiology. International Journal of Epidemiology, 36(6), 1253-1254.

Pavot, W., \& Diener, E. (1993). Review of satisfaction with life scale. Psychological Assessment, 5(2), $164-172$.

Picket, K. E., \& Pearl, M. (2001). Multilevel analyses of neighbourhood socioeconomic context and health outcomes: A critical review. Journal of Epidemiology and Community Health, 55, 111-122.

Requena, F. (2003). Social capital, satisfaction, and quality of life in the workplace. Social Indicators Research, 61(3), 331-360.

Siegrist, J. (2003). Subjective well-being: New conceptual and methodological developments in healthrelated social sciences. Working paper of the ESF SCSS exploratory workshop on 'income, interactions and subjective well-being'. http://www.delta.ens.fr/swb/SiegristSWB.pdf. Accessed Nov 2009.

Townsend, P., Phillimore, P., \& Beattie, A. (1988). Health and deprivation: Inequalities and the north. London: Croom Helm Ltd.

United Nations (2009). Human development reports 2009. Economy and inequality Gini index. http://hdrstats.undp.org/en/indicators/161.html. Accessed Nov 2009.

Veenhoven, R. (1993). Happiness in nations: Subjective appreciation of life in 56 nations 1946-1992. Rotterdam: Erasmus University Press. 
Veenhoven, R. (2000). Freedom and happiness: A comparative study in forty-four nations in the early 1990 s. In E. Diener \& M. Eunnkook (Eds.), Culture and subjective wellbeing (pp. 257-288). Cambridge, Mass: MIT Press.

Wen, M., Browning, C. R., \& Cagney, K. A. (2003). Poverty, affluence, and income inequality: Neighborhood economic structure and its implications for health. Social Science and Medicine, 57(5), $843-860$.

Wilkinson, R. G., \& Pickett, K. E. (2006). Income inequality and population health: A review and explanation of the evidence. Social Science and Medicine, 62, 1768-1784.

Winkelman, R. (2009). Unemployment, social capital, and subjective well-being. Journal of Happiness Studies, 10, 421-430.

Wong, I. O., Cowling, B. J., Lo, S. V., \& Leung, G. M. (2009). A multilevel analysis of the effects of neighbourhood income inequality on individual self-rated health in Hong Kong. Social Science and Medicine, 68(1), 124-132.

Yip, W., Sybramanian, S. V., Mitchell, A. D., Lee, D. T. S., Wang, J., \& Kawachi, I. (2007). Does social capital enhance health and well-being? Evidence from rural China. Social Science and Medicine, 64, $35-49$. 